\title{
EDITORIAL
}

Special Focus Issue: Psycho-oncology

\section{The importance of stress management among postresection breast cancer patients}
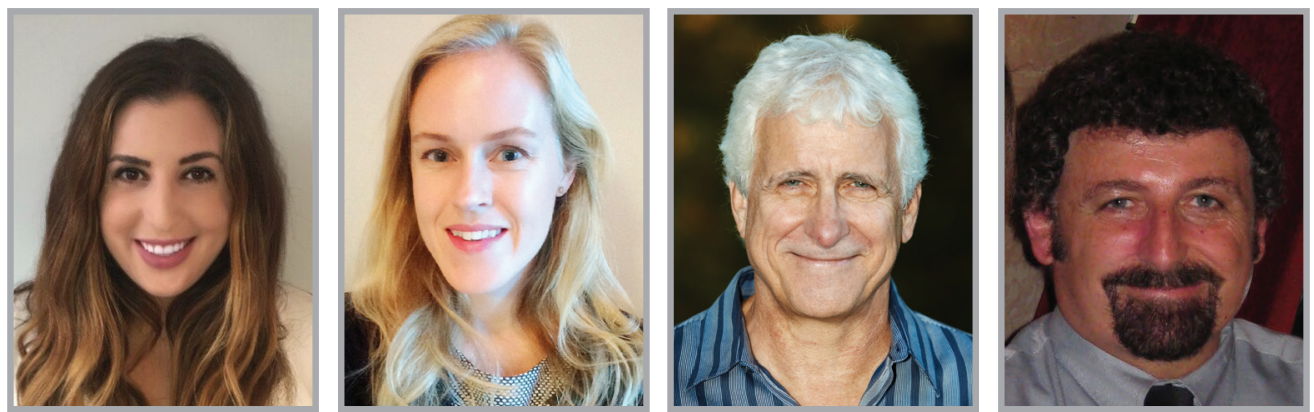

Chelsea R Amiel ${ }^{*, \neq 1}$, Hannah M Fisher**,*,1, Charles S Carver ${ }^{1,2}$

\& Michael H Antoni $\mathrm{i}^{1,2,3}$

First draft submitted: 30 September 2016; Accepted for publication: 7 October 2016; Published online: 19 October 2016

\section{Biobehavioral model: stress affects processes relevant to breast cancer progression \& clinical outcomes \\ Women with breast cancer experience sig- nificant stress throughout their illness tra- jectory, beginning in the period surround- ing diagnosis and surgery. Stress among postsurgical breast cancer (PS-BCa) patients can affect biological processes that regulate the endocrine and immune systems and these influences can have long-term effects on disease outcomes [1]. During stressful encounters, the hypo- thalamic-pituitary-adrenal (HPA) axis is activated and releases glucocorticoids (e.g., cortisol) that in turn downregulate the immune system and stabilize metabolic functions [2]. Normally, cortisol levels peak after waking and slowly decrease through the day, but if the HPA axis is chronically activated (e.g., during times of stress), cortisol levels remain high [2]. Compared}

with healthy controls, women with breast cancer exhibit higher levels of circulating cortisol and a flatter slope in their diurnal pattern, due largely to elevated evening levels [2].

Chronically elevated cortisol desensitizes immune cell glucocorticoid receptors that downregulate inflammatory responses. As a result, more inflammatory ligands (e.g., cytokines) are released into circulation [2]. Glucocorticoid receptor's resistance in circulating leukocytes can permit increased cytokine concentrations in the vicinity of tumor cells. These cytokines may contribute to disease progression by facilitating angiogenesis and endothelialto-mesenchymal transitions that promote metastasis [3]. Thus, stress-associated neuroendocrine changes may contribute to disease course in PS-BCa patients. Indeed, breast cancer recurrence and mortality have been linked to elevated cortisol prior

\section{KEYWORDS}

- breast cancer

- cognitive-behavioral stress management $\bullet$ cognitive-behavioral therapy $\bullet$ postsurgery $\bullet$ relaxation

\section{"Women with breast cancer} experience significant stress throughout their illness trajectory, beginning in the period surrounding diagnosis and surgery." 
"Postsurgical psychosocial interventions have delivered mixed results with regard to long-term clinical benefits in breast cancer patients with nonmetastatic disease." to diagnosis, as well as flatter diurnal cortisol slopes [3].

\section{Psychosocial processes that influence adaptation to breast cancer treatment}

The period after breast cancer surgery is particularly stressful, with patients often reporting heightened emotional distress, anxiety and depression; fears of recurrence, death and being damaged by adjuvant therapy and marked changes in their social world [4-6]. Ongoing intrusive thoughts about breast cancer can further exacerbate anxiety and depression, which can adversely affect quality of life and emotional functioning during treatment and survivorship [4,5]. PS-BCa patients frequently report sleep disruption and fatigue, which also affect quality of life after adjuvant therapy [7]. Generally, poorer psychosocial adaptation and quality of life during treatment predicts diminished quality of life during survivorship [4].

\section{Psychosocial interventions that improve adaptation to breast cancer treatment}

Much evidence has accrued to demonstrate the efficacy of psychosocial interventions among PS-BCa patients. Many individual and group-based interventions have been designed to address cognitions, stress appraisals and coping, using traditional cognitive behavioral therapy techniques [4]. These psychosocial interventions also frequently teach interpersonal skills (e.g., assertiveness, anger management, among others) to enhance perceptions and receipt of social support [4].

\section{Intervention effects on psychological} status \& quality of life during treatment In one randomized clinical trial (RCT), PS-BCa women assigned to a 10-week group-based cognitive-behavioral stress management (CBSM) intervention reported significant reductions in negative affect, thought intrusions and anxiety versus those in a psychoeducation control [5]. CBSM participants also reported increased positive affect, benefit finding and positive states of mind, as well as improvements in quality of life [6]. In a later CBSM 'dismantling' trial that compared the effects of 5 weeks of group-based cognitive-behavioral therapy (CBT) or relaxation training versus a 5-week group health education control condition, those assigned to CBT or relaxation training reported decreased negative affect versus controls [6]. In this trial, 5 weeks of CBT was specifically associated with reductions in breast-cancer specific intrusive thoughts.
Another trial showed that a 24-session CBT-based group intervention reduced anxiety in PS-BCa patients [8]. It is likely the shortest forms of such intervention (i.e., 5 weekly sessions) are those that are most likely to be adopted in clinical oncology practice given the limited time requirements of these approaches during the period of primary treatment [6].

CBT-based interventions ranging from 5 to 24 weeks have been shown to attenuate disturbances in social functioning and improve perceptions of social support $[4,6,8]$. A growing body of evidence has also highlighted improved health behaviors that result from psychological interventions among breast cancer patients, including improved dietary habits, reduction in smoking, increased self-efficacy in relaxation techniques and other positive lifestyle changes $[4,6,8]$.

Last, psychosocial interventions can also reduce distressing physical symptoms such as sleep, fatigue and pain that are frequently endorsed by PS-BCa women. Those assigned to CBT for insomnia reported significantly better subjective sleep quality and less use of sleep medications, as well as fewer depressive and anxious symptoms and greater general quality of life [9]. Parallel improvements in sleep quality and fatiguerelated daytime interference were observed among women who received ten sessions of CBSM versus those assigned to a psychoeducational control [7].

Intervention effects on neuroendocrine \& immune processes

A review of the field published in 2002 noted that no psychosocial intervention had been shown to clearly improve immune functioning in cancer patients [10]. That review noted poor methodology, small sample sizes and the inclusion of patients with different disease types as possible barriers to demonstrating immune effects [10]. Since the publication of that review, trials focused specifically on PS-BCa patients, have found evidence suggesting that psychosocial interventions can decrease HPA axis activity (e.g., evening serum cortisol) and increase measures of immune functioning [11].

In one RCT, PS-BCa patients assigned to 10 weeks of CBSM had decreased evening serum cortisol after the intervention and increased lymphocyte proliferative responses to challenge at 3-month follow-up compared with controls [12]. In a separate RCT, PS-BCa patients assigned to CBSM also showed significant decreases in evening serum cortisol and increases in IFN- $\gamma$ 
production from stimulated leukocytes [13], upregulation of 'antiviral' type I and II interferon signaling genes [14], and downregulation of leukocyte proinflammatory gene transcripts at 6- to 12-month follow-up [14]. Likewise, PS-BCa patients who participated in a 24-session CBTbased group-based intervention that taught stress management and encouraged positive health behaviors showed increases in lymphocyte proliferation and reductions in inflammatory indicators (e.g., white blood cell counts) $[8,15]$.

\section{Intervention effects on longer-term clinical outcomes}

Postsurgical psychosocial interventions have delivered mixed results with regard to long-term clinical benefits in breast cancer patients with nonmetastatic disease [10]. For example, even though their cognitive-existential group therapy improved psychosocial functioning (i.e., anxiety symptoms and family functioning), Kissane et al. (2004) found no survival benefit from it [16]. Patients from another trial who received 10 weeks of CBSM endorsed lower depressive symptoms and enhanced quality of life up to 15 years after breast cancer surgery versus those assigned to psychoeducation control [17]. Furthermore, women in this trial with stage $0-$ III disease exhibited a reduced risk of all-cause mortality CBSM compared with controls. Women with stage I-III disease receiving CBSM in his trial also showed lower odds of breast cancer mortality and recurrence over an 11-year median follow-up [18]. In another RCT, PS-BCa patients assigned to 24 sessions of CBT-based psychosocial intervention showed lower odds of mortality and disease recurrence over an 11-year median follow-up [19]. Thus, psychosocial interventions can not only improve short-term psychological and physiological adaptation to breast cancer, but also they may provide significant clinical benefits beyond the first decade of survivorship. One intriguing line of inquiry is now tying intervention-related downregulation of inflammatory signaling during primary treatment to a longer disease-free interval over the subsequent decade. Integrating short-term changes during primary treatment with such long-term clinical outcomes evinces the clinical significance of enhancing adaptation at the earliest points in the cancer experience.

\section{Limitations \& future directions}

While research on the impact of stress management and other psychosocial interventions for
PS-BCa women is becoming well established, there remain some methodological concerns. Psychosocial intervention research must carefully consider the potential confounding effects of individual variation in sociodemographic and disease characteristics, neuroendocrine and immunological response to surgery, hormonal treatment and adjuvant therapies [4]. Such considerations are also relevant for the timing of the intervention's delivery. Extant literature suggests that early intervention in the weeks after surgery may be an optimal time to initiate psychosocial interventions, as it represents an acute period of stress that may influence treatment response and relevant clinical outcomes [4].

More research is also needed to assess whether particularly vulnerable and at-risk populations may benefit most from postsurgical psychosocial interventions. For example, highly distressed and/or socially isolated individuals, as well as racial/ethnic minorities may respond differently to traditional CBSM or other CBT-based interventions. Initial findings on the efficacy of culturally adapted interventions have been promising in demonstrating the benefits of a culturally tailored, community-based CBSM intervention for black breast cancer survivors. More studies are necessary, however, to determine how best to serve these subpopulations during primary treatment [20]. It is plausible that other forms of behavioral intervention focused on relaxation techniques, mindfulness, yoga and physical exercise training may also bring about positive effects and these may be more or less acceptable for specific populations or at different points in treatment [4].

The studies highlighted here suggest that CBTbased psychosocial interventions may reduce stress and modify biobehavioral processes among women undergoing primary treatment for breast cancer. Ongoing work is examining specifically how intervention-associated changes in physiological markers and psychological well-being contribute to long-term clinical outcomes.

\section{Financial \& competing interests disclosure}

$M H$ Antoni reports receiving publication royalties from a book he co-authored on cognitive-behavioral stress management. The authors have no other relevant affliations or financial involvement with any organization or entity with a financial interest in or financial conflict with the subject matter or materials discussed in the manuscript apart from those disclosed.

No writing assistance was utilized in the production of this manuscript.

\section{"Cognitive-behavioral therapy-based psychosocial interventions may reduce stress and modify biobehavioral processes among women undergoing primary treatment for breast cancer."}




\section{References}

1 Andersen BL, Kiecolt-Glaser JK, Glaser R. A biobehavioral model of cancer stress and disease course. Am. Psychol. 49 (5), 389 (1994).

2 McEwen BS. Physiology and neurobiology of stress and adaptation: central role of the brain. Physiol. Rev. 87(3), 873-904, (2007).

3 Antoni MH, Lutgendorf SK, Cole SW et al. The influence of bio-behavioural factors on tumour biology: pathways and mechanisms. Nat. Rev. Cancer 6(3), 240-248 (2006).

4 Antoni MH. Psychosocial intervention effects on adaptation, disease course, and biobehavioral processes in cancer. Brain Behav. Immun. 30 (Suppl.), S88-S98 (2013).

5 Antoni MH, Wimberly S, Lechner S et al. Stress management intervention reduces cancer-specific thought intrusions and anxiety symptoms among women undergoing treatment for breast cancer. Am. J. Psychiatry 163, 1791-1797 (2006).

6 Gudenkauf L, Antoni MH, Stagl J et al. Brief cognitive-behavioral and relaxation training interventions for breast cancer: a randomized controlled trial. J. Consult. Clin. Psychol. 83(4), 677-688 (2015).

7 Vargas S, Antoni MH, Carver CS et al. Sleep quality and fatigue after stress management intervention in women under adjuvant treatment for early breast cancer. Int. J. Behav. Med. 21, 971-981 (2014).

8 Andersen BL, Farrar WB, Golden-Kreutz DM et al. Psychological, behavioral, and immune changes after a psychological intervention: a clinical trial. J. Clin. Oncol. 22, 3570-3580 (2004).

9 Savard J, Simard S, Ivers H, Morin CM. Randomized study on the efficacy of cognitive-behavioral therapy for insomnia secondary to breast cancer. Part I: sleep and psychological effects. J. Clin. Oncol. 23(25), 6083-6096 (2005)

10 Newell SA, Sanson-Fisher RW, Savolainen NJ. Systematic review of psychological therapies for cancer patients: overview and recommendations for future research. J. Natl Cancer Inst. 94(8), 558-584 (2002).

11 McGregor B, Antoni MH. Psychological intervention and health outcomes among women treated for breast cancer: a review of stress pathways and biological mediators. Brain Behav. Immun. 23, 159-166 (2009).

12 Phillips KM, Antoni MH, Lechner SC et al. Stress management intervention reduces serum cortisol and increases relaxation during treatment for nonmetastatic breast cancer. Psychosom. Med. 70, 1044-1049 (2008).

13 Antoni MH, Lechner S, Diaz A et al. Cognitive behavioral stress management effects on psychosocial and physiological adaptation in women undergoing treatment for breast cancer. Brain Behav. Immun. 23, 580-591 (2009).

14 Antoni MH, Lutgendorf S, Blomberg B et al. Cognitive-behavioral stress management reverses anxiety-related leukocyte transcriptional dynamics. Biol. Psychiatry 71 , 366-372 (2012).

15 Thornton LM, Andersen BL, Schuler TA, Carson WE III. A psychological intervention reduces inflammatory markers by alleviating depressive symptoms: secondary analysis of a randomized controlled trial. Psychosom. Med. 71(7), 715-724 (2009).

16 Kissane DW, Love A, Hatton A et al. Effort of cognitive-existential group therapy on survival in early-stage breast cancer. J. Clin. Oncol. 22(21), 4255-4260 (2004).

17 Stagl J, Bouchard L, Lechner SC et al. Long term psychological benefits of cognitive-behavioral stress management for women with breast cancer: 11-year follow-up of a randomized controlled trial. Cancer 121, 1873-1881 (2015).

18 Stagl JM, Lechner SC, Carver CS et al. A randomized controlled trial of cognitive-behavioral stress management in breast cancer: survival and recurrence at 11-year follow-up. Breast Cancer Res. Treat. 154(2), 319-328 (2015).

19 Andersen BL, Yang HC, Farrar WB et al. Psychologic intervention improves survival for breast cancer patients. Cancer 113(12), 3450-3458 (2008)

20 Lechner SC, Whitehead NE, Vargas S et al. Does a community-based stress management intervention affect psychological adaptation among underserved black breast cancer survivors? J. Natl Cancer Inst. Monogr. 50, 315-322 (2014). 